\section{Forschungspreise der DGP 2016}

Die Deutsche Gesellschaft für Pneumologie und Beatmungsmedizin e.V. (DGP) schreibt für das kommende Jahr 2 Forschungspreise aus. Die Preise dienen der Förderung des wissenschaftlichen Nachwuchses in der Pneumologie. Sie werden für die beste grundlagenwissenschaftliche und die beste klinische Arbeit aus dem Gesamtgebiet der Pneumologie verliehen und sind mit jeweils 10000 Euro dotiert.

Die eingereichte Arbeit muss in deutscher oder englischer Sprache verfasst und darf zu keinem anderen Preis eingereicht sein. Ist die Arbeit bereits publiziert, darf das Erscheinungsdatum der Publikation nicht mehr als 1 Jahr vor dem Abgabetermin zur Preiseinreichung liegen. Mehrere thematisch zusammenhängende Publikationen können zusammengefasst und (mit einem gemeinsamen Abstract versehen) eingereicht werden. Sollte die Arbeit in einer Forschergruppe erstellt oder der Bewerber nicht Erstautor einer zugehörigen Publikation sein, bitten wir um Angabe des Eigenanteils.

Die Bewerber sollen zum Zeitpunkt der Bewerbungsfrist nicht über 40 Jahre alt sein. Sie müssen ihre Arbeitsstätte in Deutschland haben. Wenn die Forschungsarbeiten für die eingereichten Veröffentlichungen in Deutschland ausgeführt wurden, wird dies ebenfalls berücksichtigt.

Beide Preise werden auf der Eröffnungsveranstaltung des DGP-Kongresses am 2. März 2016 in Leipzig verliehen.

Die Bewerber werden gebeten, ihre Arbeit bis zum 8. Januar 2016 im PDF-Format (max. $5 \mathrm{MB}$ ) mit einer Publikationsliste und ihrem Lebenslauf an den Generalsekretär der DGP, Prof. Felix J. Herth, (felix.herth@med.uni-heidelberg.de) sowie an office@dgpberlin.de zu schicken. Unvollständige Bewerbungen werden nicht berücksichtigt. Die Entscheidung des Auswahlkomitees ist nicht anfechtbar.

Nach einer Mitteilung der DGP, Berlin

\title{
Genetischer Zusammenhang mit erhöhtem Diabetesrisiko
}

\author{
Die chronisch obstruktive Atemwegserkrankung (COPD) führt \\ zu hoher Morbidität und Mortalität und ist mit zahlreichen, \\ systemischen Erkrankungen assoziiert - auch mit Diabetes \\ mellitus Typ 2. Die dänische Arbeitsgruppe um H. Meteran \\ ging nun der Hypothese nach, ob dies auf gemeinsamen \\ genetischen Faktoren beider Erkrankungen beruhen könnte. \\ Respir Med 2015; 109: 1026-1030
}

Dazu analysierten die Forscher die Daten von 13649 Zwillingspaaren aus der dänischen Zwillingskohorte mit vollständigen Daten zu chronischer Bronchitis und Rauchstatus und verknüpften sie mit Daten zu Diagnosen bei Krankenhausentlassung aus einem entsprechenden nationalen Register. Das Alter der Zwillinge lag zwischen 50 und 71 Jahren, 33\% waren aktuelle Raucher, 30\% ehemalige Raucher. Anhand der dokumentierten Diagnoseschlüssel identifizierten die Autoren 1160 Personen mit chronischer Bronchitis (Prävalenz in der Kohorte: 8,9\%), 380 mit COPD (Prävalenz 2,8\%) und 332 mit insulinabhängigem Diabetes mellitus Typ 2 (Prävalenz 2,4\%).

\section{Deutliche Assoziationen \\ $\nabla$}

Das Risiko für einen Typ 2-Diabetes war bei Personen mit chronischer Bronchitis höher als bei solchen ohne diese Symptome (3,5 vs. 2,3\%; Odds Ratio [OR] 1,57; 95\%-Konfidenzintervall [KI] 1,10-2,26; $\mathrm{p}=0,014)$. Bei COPD-Diagnose war die Assoziation mit Diabetes mellitus Typ 2 im Vergleich zu Personen ohne eine solche Diagnose oder Bronchitissymptome noch etwas stärker (6,6 vs. 2,3\%; OR 2,62; 95\%-KI 1,63-4,2; $\mathrm{p}<0,001)$. Auch unter Berücksichtigung anderer Einflussfaktoren wie Alter, Geschlecht, Rauchstatus und Body Mass Index (BMI) blieben diese Assoziationen statistisch signifikant.

Die Konkordanzrate von chronischer Bronchitis und Diabetes war bei eineiigen Zwillingen etwas höher als bei zweieiigen, bei COPD und Diabetes aber etwa vergleichbar. Die Varianzkomponenten-
Analyse zeigte eine Heredität von $44 \%$ bei chronischer Bronchitis, $43 \%$ bei COPD und $57 \%$ bei Diabetes. Die Korrelation der genetischen Effekte auf chronische Bronchitis und Diabetes Typ 2 betrug 0,33 (95\%-KI 0,00-0,79; $\mathrm{p}=0,103$ ), die von COPD und Diabetes 0,43 (95\%-KI 0,00$0 ; 98 ; p=0,154)$. Dass die Korrelationen nicht signifikant wurden, führen die Autoren auf die relativ kleine Zahl von Diagnosen, insbesondere eines Diabetes mellitus Typ 2 zurück. Sie sehen eher einen gemeinsamen kausalen Effekt (z.B. eine systemische Inflammation) als Ursache für eine erhöhte Suszeptibilität für COPD und Diabetes an als eine gemeinsame genetische Ursache der Komorbidität.

Fazit
Patienten mit chronischer Bronchitis
oder COPD haben unabhängig von ande-
ren Einflussfaktoren ein erhöhtes Risiko,
an Diabetes mellitus zu erkranken. Die
genetische Korrelation zwischen Diabe-
tes und chronischer Bronchitis lag bei
$33 \%$, die von COPD und Diabetes bei $43 \%$,
erreichte aber keine statistische Signifi-
kanz. In der Praxis sollte das erhöhte Di-
abetesrisiko bei der Versorgung von Pa-
tienten mit chronischer Bronchitis und
COPD berücksichtigt werden, so die Au-
toren.

Friederike Klein, München 